\title{
The inflated mitochondrial genomes of siphonous green algae reflect processes driving expansion of noncoding DNA and proliferation of introns
}

\author{
Sonja I Repetti ${ }^{\text {Corresp., }}{ }^{2}$, Christopher J Jackson ${ }^{1}$, Louise M Judd ${ }^{2}$, Ryan R Wick ${ }^{2}$, Kathryn E Holt ${ }^{2}$, Heroen \\ Verbruggen $^{1}$ \\ ${ }^{1}$ School of BioSciences, University of Melbourne, Melbourne, Victoria, Australia \\ 2 Department of Infectious Diseases, Monash University, Melbourne, Victoria, Australia \\ Corresponding Author: Sonja I Repetti \\ Email address: sonjarepetti@live.com.au
}

Within the siphonous green algal order Bryopsidales, the size and gene arrangement of chloroplast genomes has been examined extensively, while mitochondrial genomes have been mostly overlooked. The recently published mitochondrial genome of Caulerpa lentillifera is large with expanded noncoding DNA, but it remains unclear if this is characteristic of the entire order. Our study aims to evaluate the evolutionary forces shaping organelle genome dynamics in the Bryopsidales based on the C. lentillifera and Ostreobium quekettii mitochondrial genomes. In this study, the mitochondrial genome of $O$. quekettii was characterised using a combination of long and short read sequencing, and bioinformatic tools for annotation and sequence analyses. We compared the mitochondrial and chloroplast genomes of $O$. quekettii and $C$. lentillifera to examine hypotheses related to genome evolution. The $O$. quekettii mitochondrial genome is the largest green algal mitochondrial genome sequenced $(241,739 \mathrm{bp})$, considerably larger than its chloroplast genome. As with the mtDNA of $C$. lentillifera, most of this excess size is from the expansion of intergenic DNA and proliferation of introns. Inflated mitochondrial genomes in the Bryopsidales suggest effective population size, recombination and/or mutation rate, influenced by nuclear-encoded proteins, differ between the genomes of mitochondria and chloroplasts, reducing the strength of selection to influence evolution of their mitochondrial genomes. 
1 The inflated mitochondrial genomes of siphonous green algae reflect processes driving

2 expansion of noncoding DNA and proliferation of introns

3 Sonja I. Repetti ${ }^{1}$, Christopher J. Jackson ${ }^{1}$, Louise M. Judd ${ }^{2}$, Ryan R. Wick ${ }^{2}$, Kathryn E. Holt ${ }^{2}$,

$4 \quad$ Heroen Verbruggen ${ }^{1}$

$5 \quad{ }^{1}$ School of BioSciences, University of Melbourne, Melbourne, Victoria, Australia

$6{ }^{2}$ Department of Infectious Diseases, Monash University, Melbourne, Victoria, Australia

8 Corresponding Author:

9 Sonja Repetti ${ }^{1}$

Email address: sonjarepetti@live.com.au

11

12

13

14

15 


\section{Abstract}

Within the siphonous green algal order Bryopsidales, the size and gene arrangement of chloroplast genomes has been examined extensively, while mitochondrial genomes have been mostly overlooked. The recently published mitochondrial genome of Caulerpa lentillifera is large with expanded noncoding DNA, but it remains unclear if this is characteristic of the entire order. Our study aims to evaluate the evolutionary forces shaping organelle genome dynamics in the Bryopsidales based on the C. lentillifera and Ostreobium quekettii mitochondrial genomes. In this study, the mitochondrial genome of $O$. quekettii was characterised using a combination of long and short read sequencing, and bioinformatic tools for annotation and sequence analyses. We compared the mitochondrial and chloroplast genomes of $O$. quekettii and C. lentillifera to examine hypotheses related to genome evolution. The $O$. quekettii mitochondrial genome is the largest green algal mitochondrial genome sequenced $(241,739 \mathrm{bp})$, considerably larger than its chloroplast genome. As with the mtDNA of $C$. lentillifera, most of this excess size is from the expansion of intergenic DNA and proliferation of introns. Inflated mitochondrial genomes in the Bryopsidales suggest effective population size, recombination and/or mutation rate, influenced by nuclear-encoded proteins, differ between the genomes of mitochondria and chloroplasts, reducing the strength of selection to influence evolution of their mitochondrial genomes.

\section{Introduction}

Although they share many unifying features, the green algae (Chlorophyta) are a diverse group with genomes that vary considerably in structure and gene content (Yurina \& Odintsova, 2016). Most work on Chlorophyta genomes to date has focused on organellar genomes, with in excess 
42 of 150 chloroplast and 70 mitochondrial genomes published on Genbank. These organelle

43

44 genome data have been used for phylogenetic analyses to resolve lineage relationships within the Chlorophyta (e.g. Fučíková et al., 2014; Cremen et al., 2018) and to investigate organellar genome structural adaptation and unique features. As with many plastid-bearing taxa, more effort has been made to sequence and characterise chloroplast genomes rather than mitochondrial, as plastid genomes tend to be favoured for their use in phylogenetic studies due to high coding content, relatively conserved genome and simple inheritance patterns (Smith \& Keeling, 2015; Fang et al., 2017). There are some notable exceptions in the Chlorophyta such as the fragmented hairpin plasmids seen in Cladophorales plastids (Del Cortona et al., 2017) and the large and highly repetitive Acetabularia acetabulum (Dasycladales) plastid genome (de Vries et al., 2013). Despite this, mitochondrial genomes tend to show greater abnormalities in structure than plastid genomes (Burger et al., 2003), and some of the most unusual genomic features have been observed in eukaryotic mitochondrial genomes (Smith \& Keeling, 2015).

Comparative studies in green algal chloroplast and mitochondrial genomes have found that many instances of genome size variation are not due to gene gain or loss, but rather reduction or expansion of non-coding DNA including introns (Burger et al., 2003; Smith et al., 2013; Marcelino et al., 2016). In the Chlamydomonadales, Smith et al. (2013) observed an increase in chloroplast DNA from approximately $60 \%$ noncoding DNA in smaller-sized unicellular lineages to greater than $80 \%$ in multicellular Volvox carteri, while the number of genes differs by only two between the smallest and largest genomes. A similar pattern was observed for their mitochondrial DNA (Smith et al., 2013). According to Smith and Lee (2010), the large noncoding DNA content in $V$. carteri genomes could support the mutational-hazard hypothesis (MHH). The MHH proposes that excess DNA is more likely to accumulate in genomes with a 
65 low mutation rate and small effective population size $\left(N_{e}\right)$ (Lynch, 2006; Smith, 2016) due to

66

67 non-coding DNA imposing a fitness burden by increasing the potential for harmful mutations. Such excess DNA can be eradicated more effectively when there is a larger effective population size for natural selection to act upon through purifying selection (Lynch \& Walsh, 2007). The MHH might explain the streamlining of organelle genomes within various lineages including prasinophytes, red algae, and some fungi, which have high estimated mutation rates (Smith, 2016). A large reduction in $N_{e}$ is expected for multicellular organisms compared with unicellular, therefore purifying selection should be less efficient meaning there is less restriction on the accumulation of noncoding DNA. This may explain the difference in noncoding content between unicellular members of the Chlamydomonadales, such as Chlamydomonas, and colonial $V$. carteri (Smith et al., 2010). Various studies have supported and challenged the MHH (see Smith, 2016), but on the whole emphasise the importance of mutation and genetic drift in shaping organelle genomes (Lynch, 2006; Smith, 2016).

It has also been suggested that selection can influence the mutation rate of genomes. The driftbarrier hypothesis (Lynch et al., 2016) proposes that selection acts to reduce the mutation rate with an overall limit set by genetic drift. In genomes with very high mutation rates, 'antimutators' such as DNA repair proteins are advantageous and decrease the mutation rate until the strength of selection is matched by that of genetic drift and mutation bias (Lynch et al., 2016). In contrast, in genomes with low mutation rates, antimutators will not be advantageous enough to be selected for, whilst having mild mutators will not be sufficiently disadvantageous to be selected against. Therefore, mutation rate will increase until selection is strong enough to prevent the genome evolving a higher mutation rate (Lynch et al., 2016). Krasovec et al. (2017) combined a review of literature with mutation rate estimates for four prasinophytes and found 
88 support for this theory, with mutation rate tending to decrease as $N_{e}$, therefore strength of

89 selection, increases.

90 The effective population size of genomes influences the efficacy of selection relative to genetic

91 drift (Ness et al., 2015). Within genomes, loci linked to sites under selection will exhibit locally

92 reduced $N_{e}$ due to hitchhiking, where mutations that are not necessarily optimal are fixed because

93 they are located close to another gene undergoing selection (Smith \& Haigh, 1974), background

94 selection, when selection acts against linked deleterious alleles that are retained in a population

95 by mutation (Charlesworth,1994), and Hill-Robertson (HR) interference (Platt et al., 2018 and

96 references therein). Under the HR effect (Hill \& Robertson, 1966), weak selection acting on

97 linked sites reduces the overall effectiveness of selection due to linkage disequilibrium (Comeron

98 et al., 2008). Recombination breaks up such linkage and prevents local reduction in $\mathrm{N}_{\mathrm{e}}$ due to

99 selection acting upon linked sites (Cutter \& Payseur, 2013; Platt et al., 2018). It has been

100 observed that genes located in regions of low recombination have a greater number of introns,

101 and these introns have greater lengths (Comeron \& Kreitman, 2000). It has been proposed that

102 introns may be advantageous in reducing intragenic HR effect and are hence retained, as they

103 might increase rates of recombination that lower intragenic HR effects (Comeron et al., 2008).

104 Comeron \& Kreitman (2000) suggest that longer introns are advantageous in regions of low

105 recombination, as they reduce the HR effect relative to a shorter intron by increasing the

106 recombination rate between mutations in different exons, and that they will be maintained due to

107 hitchhiking effects between favourable mutations and the longest intron variant.

108 The class Ulvophyceae within the Chlorophyta show high morphological diversity, including a

range of cell configurations (Cocquyt et al., 2010; Fang et al., 2017), and are thus a useful model

110 for examining the evolution of genome features under differing evolutionary constraints. The 
111 order Bryopsidales are a lineage of siphonous seaweeds with thali comprised of a single giant

112 tubular cell containing cytoplasm with a large number of nuclei and other organelles free to

113 move around the entire plant (Vroom \& Smith, 2001; Vroom \& Smith, 2003; Verbruggen et al.,

114 2009; Mine et al., 2015). Within the Bryopsidales, the size and gene arrangement of chloroplast

115 genomes has been found to vary considerably while gene content was much less variable

116 (Cremen et al., 2018). While appreciable short-read sequencing data have been generated for the

117 Bryopsidales, mitochondrial genomes have not been found to assemble well from the sequencing

118 libraries used to assemble chloroplast genomes, and the first bryopsidalean mitochondrial

119 genome was published only very recently for the sea grape Caulerpa lentillifera (Zheng et al.,

120 2018). This 209,034 bp circular-mapping genome is the largest Chlorophyta mitochondrial

121 genome published to date, an order of magnitude larger than the average for Chlorophyta

mitochondrial genomes and rich in intergenic sequences and introns, the causes of which have not been examined.

Unlike the genome expansion observed in the Chlamydomonadales, which appears to occur in

both organellar compartments (Smith et al., 2013; Zhang et al., 2019), C. lentillifera's

mitochondrial genome is much larger than its chloroplast genome. This is unusual for green algae where mitochondrial genomes are generally smaller than chloroplast genomes (Leliaert et al., 2012). Furthermore, mitochondrial genome expansion is less frequent in green algae compared with plants (Mower et al., 2012). While Zheng et al. (2018) did not propose an evolutionary explanation for these findings, the inflated noncoding regions described are consistent with previous studies in plants showing that the variation in the sizes of their mitochondrial genomes is predominantly explained by changes in noncoding DNA content including repeats, introns, intergenic DNA and DNA of foreign origin (Yurina \& Odintsova, 
2016). Therefore, the mitochondrial genome of $C$. lentillifera provides an interesting case in the Chlorophyta to examine the evolution of organellar genomes and, in particular, the processes driving genome expansion. However, it remains undetermined whether a large mitochondrial genome is a feature only of Caulerpa, or if it is in fact characteristic of the order Bryopsidales.

In this paper, we focus on another member of the Bryopsidales that is currently of considerable ecological interest: the genus Ostreobium, an endolithic limestone-boring alga (Verbruggen \& Tribollet, 2011). Ostreobium is found in a diverse range of calcium carbonate environments around the world, is abundant in the skeleton of many coral species, and it is one of the most common genera of endolithic autotrophs in coral reefs (Tribollet, 2008). Endolithic algae like Ostreobium have a number of observed and predicted roles in the coral skeleton (summarised in Ricci et al., 2019). While their boring can destabilise coral skeletons and impact on reef structure (Schlichter et al., 1997), they may have a mutualistic relationship with corals, providing them with metabolites that could enable them to survive bleaching events (Schlichter et al., 1997; Tribollet, 2008). Its endolithic lifestyle means that Ostreobium inhabits low light habitats with limited available photosynthetically active radiation (Wilhelm \& Jakob, 2006; Magnusson et al., 2007) and it has been shown to have far red-shifted absorption spectra, allowing it to use the long wavelengths of light available in the coral skeleton (Wilhelm \& Jakob, 2006; Magnusson et al., 2007; Tribollet, 2008).

Ostreobium species have consistently small chloroplast genomes relative to the median for Bryopsidales of 105kb (Cremen et al., 2018), with the chloroplast genome of Ostreobium strain HV05042 the most compact found so far (80,584 bp) in the Ulvophyceae (Marcelino et al., 2016; Verbruggen et al., 2017). Marcelino et al. (2016) identified only three introns in the O. quekettii chloroplast genome, and there was an overall reduction in intergenic regions that resulted in its 
157 reduced size. They hypothesized that this might reflect evolution in response to resource 158 constraints in its low light environment (Marcelino et al., 2016), but did not examine the 159 mitochondrial genome for evidence of similar selection pressures.

160 The goal of this study is to evaluate the characteristics of mitochondrial genomes in the

161

162 163 
179 were filtered using Filtlong (https://github.com/rrwick/Filtlong), removing reads less than

$1801000 \mathrm{bp}$ in length, with average quality of less than 60 (as defined by the Phred scores), or

181 minimum quality over a sliding window of less than 40.

182

183

184

185

186

187

188

189

190

191

192

193

194

195

196

197

200

201

\section{Genome assembly}

The Illumina data were assembled using Spades v3.12.0 (Bankevich et al., 2012) with the -careful option. The Nanopore data were assembled using Canu 1.7.1 (Koren et al., 2017) with the parameters genomeSize $=300 \mathrm{~m}$ corOutCoverage $=10000$ corMhapSensitivity $=$ high corMinCoverage $=0$.

A hybrid (combined long-read and short-read) genome assembly was performed with MaSuRCA v3.2.7 (Zimin et al., 2013), using the Illumina reads as short-read input. For long-read input, contigs produced by Spades were treated as 'pseudo-Nanopore' reads and combined with Nanopore data, and this combined dataset was used.

\section{Transcriptome}

Total RNA was extracted using Plant RNA reagent (Thermofisher). A strand-specific 100 bp paired-end library was constructed and sequenced using Illumina HiSeq 2500 (ENA study accession number PRJEB35267). Quality filtering of reads was performed using Trimmomatic 0.39 (Bolger et al., 2014) with the following settings: LEADING:3

TRAILING:3 SLIDINGWINDOW:4:20. A transcriptome was constructed using Trinity version 2.8.3 (Grabherr et al., 2011).

\section{Identification and manual curation of mitochondrial genome}

The Caulerpa lentillifera mitochondrial genome (Genbank accession KX761577.1) (Zheng et al., 2018) was used as the query in a BLASTn search against the long-read Canu assembly within Geneious version 11.1.2 (Kearse et al., 2012) with default settings. Only a single contig 
202 was identified as a likely candidate for the $O$. quekettii mitochondrial genome based on the

203 results of BLASTx searches of sections of this candidate contig against the NCBI nr and nt

204 databases.

205 This contig was used as the query in a BLASTn search against the O. quekettii Spades short-read

206 assembly, within Geneious with default settings. Top hits were aligned with the long-read contig

207 within Geneious. Scaffolds from the short-read assembly were used as a reference to manually

208 correct the long-read contig within Geneious. In order to verify that the genome was circular-

209 mapping, we searched for a short-read scaffold spanning across both ends of the long-read contig

210 when aligned.

211 This manual result was compared with the mitochondrial contig identified, through blast

212 searches against the NCBI nr and nt databases, in the MaSuRCA hybrid assembly by alignment

213 in Geneious.

214 Genome annotation

215 The genome was initially annotated using MFannot (Beck \& Lang, 2010), and DOGMA

216 (Wyman et al., 2004) with very relaxed settings (protein identity cut off $25 \%$, RNA identity cut 217 off $30 \%$ ). Annotations of predicted protein coding genes were confirmed through extraction of 218 open reading frames and BLAST searches of these against the NCBI nr and nt databases, as well 219 as alignment with transcripts that were recovered as hits from BLASTn searches against the $O$. 220 quekettii transcriptome within Geneious, using default settings.

221 tRNAs were identified using tRNAscan-SE (Lowe \& Chan, 2016), tRNAfinder (Kinouchi \& 222 Kurokawa, 2006), ARAGORN (Laslett \& Canback, 2004) and tRNADB-CE’s BLASTN/Pattern 223 Search (Abe et al., 2010). rRNAs were identified with RNAmmer (Lagesen et al., 2007) and 224 RNAweasel (Lang et al., 2007). 
225 Repeats were identified using the tandem repeats database (Gelfand et al., 2006), the

226 RepeatFinder package in Geneious with a minimum repeat length of $50 \mathrm{bp}$ (as per Pombert et al., 227 2004) and REPuter (Kurtz et al., 2001) with minimal repeat size setting of 12 bp (as per Smith \& 228 Lee, 2009). Forward, reverse, complement, and reverse complement repeats were all considered 229 under REPuter

230 A map of the genome was created with Circos (Krzywinski et al., 2009) and manually annotated 231 in Inkscape 0.92 (www.inkscape.org). The sequence for the Ostreobium quekettii mitochondrial 232 genome is available on Genbank (Accession number MN514984).

\section{Open reading frames}

234

235

236

237

Open reading frames (ORFs) were predicted using ORF finder in Geneious with a minimum length setting of $300 \mathrm{bp}$. These were used as queries in BLASTx searches against the NCBI nr and nt databases $\left(\mathrm{e}\right.$ value $\left.=\mathrm{e}^{-1}\right)$, and the translated ORFs were used as queries in a batch sequence search against the Pfam database (Finn et al., 2016). Only ORFs that had BLAST results and identified Pfam domains were retained in the final genome annotation. In order to determine relationships between ORFs indicative of common origin, the annotated ORFs along with those from the chloroplast genome of O. quekettii (Marcelino et al., 2016) and the mitochondrial genome of C. lentillifera (Zheng et al., 2018) were clustered based on all-againstall BLAST+ similarities using CLANs (Frickey \& Lupas, 2004). This was performed through the MPI Bioinformatics toolkit (Zimmermann et al., 2018), with the BLOSUM62 scoring matrix and extraction of BLAST HSPs up to e-values of 1e-4. The output from CLANs was annotated in Inkscape.

\section{Introns}

Intron boundaries were inferred by aligning predicted genes with their corresponding transcripts 
248 and intron-lacking homologues from other green algae. Intron class was predicted using

249 RNAweasel, and Rfam sequence search (Griffiths-Jones et al., 2003).

250 In order to identify potential common origins of introns in the $O$. quekettii and C. lentillifera 251 mitochondrial genomes as well as the $O$. quekettii plastid genome, we used a distance-based 252 clustering technique. In contrast to the analysis of ORFs above, this analysis used DNA 253 sequences and used introns that were not interrupted by ORFs, in the hope that this would 254 represent a subset of the data that could be more easily aligned if they contained conserved

regions. From the intronic DNA sequences, a distance matrix was constructed by comparing the introns from these genomes using Clustal Omega (Sievers et al., 2011) with the '--distmat-out' and '--full' flags. This distance matrix was used as the input to construct a neighbour joining (NJ) tree using Neighbor within the PHYLIP package (Felsenstein, 2005). We also constructed a distance matrix using a subset of these introns from only the $O$. quekettii chloroplast and mitochondrial genomes and this was used to construct a neighbour joining tree. This neighbour joining tree was further visualised and annotated in MEGA (Kumar et al., 2018). Clusters of introns identified in the neighbour joining tree were aligned in Geneious using MAFFT to better evaluate similarity. Insertion sites of introns were also compared between the O. quekettii and $C$. lentillifera genomes by aligning gene sequences in Geneious using MAFFT.

\section{Rates of evolution}

To obtain an estimate of the relative rates of evolution in the $O$. quekettii mitochondrial and chloroplast genomes, we aligned all the protein coding and rRNA genes common between the $O$. quekettii and C. lentillifera mitochondrial and chloroplast genomes in Geneious using the default aligner. We generated estimates of base substitutions per site between sequences using the Jukes- 
270 Cantor model (Jukes \& Cantor, 1969) in MEGA. We also attempted to obtain $d_{N} / d_{S}$ ratios for

271 gene alignments using PAL2NAL (Suyama et al., 2006).

272 Mitochondrion-targeted nuclear genes

273 In the context of evaluating hypotheses around mitochondrial DNA repair machinery (see

274 below), we searched for homologues of nuclear-encoded DNA repair proteins that are targeted to

275 the mitochondrion in higher plants: MSH1, RECA proteins and OSB1. Arabidopsis sequences

276 were used as queries in BLAST searches with default settings against the nuclear contigs in the

277 O. quekettii hybrid assembly, as well as the O. quekettii transcriptome in Geneious. We searched

278 for putative targeting signals to the mitochondrion with DeepLoc-1.0 using default settings

279 (Almagro Armenteros et al., 2017).

\section{Results}

282

283

284

285

286

287

288

289

290

291

292

\section{Ostreobium Mitochondrial Genome}

The mitochondrial genome was captured by a single contig in the Canu assembly, but six separate contigs in the Spades assembly. The genome was captured by a single contig in the hybrid genome assembly that showed $99.97 \%$ pairwise identity with our manually corrected version of the genome. The combination of long- and short-read data improved this assembly and allowed us to capture the entire genome with a high level of accuracy.

The mitochondrial genome of $O$. quekettii SAG6.99 assembled into a 241,739 bp circularmapping molecule (Fig. 1). All 64 codons are used (Table S1) and the 28 tRNAs encoded by the O. quekettii mtDNA (Table S2) appear to be sufficient to recognise all of these codons assuming the standard genetic code and maximum use of wobbling and superwobbling (Alkatib et al., 2012). Only $25 \%$ of the genome is coding DNA (Table 1 ). The genome encodes 3 rRNAs and 28 
tRNAs (Table 2, 3), resembling other green algal mitochondrial genomes (Table S3). It also encodes 34 named protein-coding genes commonly found in green algae (Table 3, S3). This does include some genes that are less common in Chlorophyta mitochondrial genomes: nad10, which is absent from the mtDNA of sequenced land plants and many green algae (Mower et al., 2012), and tatC, a gene encoding a component of the inner membrane TAT translocase responsible for transporting folded proteins across the membrane in bacteria but whose function in mitochondria remains unclear (Carrie et al., 2016; Petrů et al., 2018).

The overall GC content of the genome is $48.3 \%$ (Table 1), which is higher than the average for eukaryotic mitochondrial genomes (38\%) (Smith \& Lee, 2007) but similar to that of $C$. lentillifera (50.9\%) (Zheng et al., 2018) and not extreme for green algae (Del Vasto et al., 2015). It does however vastly exceed the GC content of the $O$. quekettii chloroplast genome (31.9\%) (Marcelino et al., 2016).

The $O$. quekettii mitochondrial genome contains 373 repeats, found in both introns and intergenic DNA, that represent $5 \%$ of the total genome (Fig. 1, Table 1), with a minimum length of 31 and maximum of 299 bp (mean 107.3 $\pm 61.7 \mathrm{SD}$ ). This is similar to the mitochondrial genome of $C$. lentillifera, where we estimated 349 repeats represent approximately $4 \%$ of the genome, with a minimum length of $25 \mathrm{bp}$ and maximum of $163 \mathrm{bp}$ (mean $68.3 \pm 30.1 \mathrm{SD}$ ).

\section{Introns}

Most of the genome's inflated size $(75 \%)$ is from expanded intergenic and intronic regions rather than extra coding material. 18 of the 34 named protein-coding genes contain one or multiple intron(s) (Table 2, S4). Introns include both type I and type II introns, as well as five whose class could not be confidently determined (Table 2, S4). The Ostreobium mitochondrial genome contains 47 introns, compared with only 29 in the mtDNA of $C$. lentillifera (Zheng et al., 2018). 
316 Type II introns, which appear to have been abundant early in the evolution of Bryopsidales

317 plastid genomes (Cremen et al., 2018) and are the dominant type in plant mtDNA but less

318 common elsewhere (Lang et al., 2007), are the dominant type in the O. quekettii mtDNA. This

319 contrasts with the $C$. lentillifera mitochondrial genome, which also contains both types of introns

320 but with type I introns predominating (Zheng et al., 2018).

ORF-lacking introns from the $O$. quekettii and $C$. lentillifera mitochondrial genomes mostly form separate clusters in neighbour joining (NJ) trees of pairwise DNA distances (Fig. S1).

Alignments of the few introns that do cluster together did not show convincing homology

between $C$. lentillifera and $O$. quekettii mitochondrial genomes (data not shown), nor between introns in the O. quekettii mitochondrial and plastid genomes (Fig, S2). Furthermore, comparing insertion sites for introns between $O$. quekettii and $C$. lentillifera only revealed five sites where intron insertion sites of the same type of intron were within $3 \mathrm{bp}$ of each other, and only a single site where they matched exactly (Table S4). The NJ tree did identify groups of similar type II introns within the $O$. quekettii mitochondrial genome, and these sequences did show convincing similarity at the DNA level in alignments, suggesting that these groups of introns proliferated within the Ostreobium lineage (Fig. S2).

ORFs with identifiable Pfam domains were only found in introns, not intergenic DNA (Table 2). Eighteen of the introns in the $O$. quekettii mitochondrial genome contain one or more ORFs. In a similarity network generated from all against all BLAST + similarities, these ORFs appear to cluster together based on shared Pfam domains, which predict similar functions for these ORFs (Fig. 2). ORFs show homology to intron-encoded proteins that act as maturases and homing endonucleases, which enable splicing and promote intron mobility (Lambowitz \& Belfort, 1993; Lambowitz \& Zimmerly, 2011; Hausner, 2012). They contain a variety of domains, but mostly 
339

340

341

342

343

344

345

346

347

348

349

350

351

352

353

354

355

356

357

358

359

360

two types of domains with the amino-acid motif LAGLIDADG, which are common in both group I and group II introns (Hausner, 2012). Three ORFs contain double LAGLIDADG 1

domains, which in other lineages are in intron-encoded proteins with maturase activity that enable intron splicing (Lambowitz \& Belfort, 1993). Four ORFs contain an RVT domain;

Cremen et al. (2018) found evidence for the mobility of group II intron-encoded ORFs containing an RVT domain within Bryopsidales chloroplast genomes.

Only one ORF identified by Zheng et al. (2018) in C. lentillifera had a positive hit to the Pfam database: ORF932 in cox 1 had a putative LAGLIDADG_1 domain. This sequence clustered with O. quekettii ORF sequences containing the same domain (Fig. 2).

None of the $O$. quekettii mitochondrial ORFs show similarity to the single ORF460 identified in the O. quekettii chloroplast, which has a putative intron splicing function (Cremen et al., 2018). Along with the results for the introns, this suggests there is little evidence for the transfer of ORFs or introns between organellar genomes in $O$. quekettii.

\section{Testing evolutionary theories: rates of evolution and mitochondrion-targeted proteins}

The inflated mitochondrial genome of $O$. quekettii prompted several hypotheses (see below) that could be examined using molecular data.

In order to gain insight into whether there are differential mutation rates between the plastid and mitochondrial genomes, that might help explain their differing sizes in both $O$. quekettii and $C$. lentillifera, we aligned genes from the two organellar genomes between the two species.

Estimates of base substitutions per site using a simple Jukes-Cantor model showed a slightly higher $(\mathrm{t}=2.94, \mathrm{p}<0.01)$ number of average base substitutions per site for the mitochondrial genomes (mean $0.456 \pm 0.207 \mathrm{SE}$ ) compared with chloroplast genomes (mean $0.370 \pm 0.016 \mathrm{SE}$ ) 
361 (Table S5). Genes showed signs of saturated divergence, with estimated $d_{S}$ values considerably

362 greater than one (e.g. atp6 $\left.d_{S}=46.8711\right)$.

363 To identify potential homologues of nuclear-encoded DNA repair proteins that are targeted to the 364 mitochondrion in higher plants and may play a role in organellar genome maintenance and size 365 (see below), we performed blast searches of Arabidopsis sequences against the $O$. quekettii 366 transcriptome and nuclear contigs. We identified a sequence in $O$. quekettii with strong similarity 367 to the Arabidopsis DNA mismatch repair protein MSH1 and putative targeting to the 368 mitochondrion (DeepLoc-1.0L: Mitochondrion 0.7151, Soluble 0.6145). Pfam and InterPro 369 searches identified a putative specific DNA-binding GIY-YIG domain in this MSH1 homologue, 370 as well as in potential homologues in other green algae. However, this domain lacks most of the key residues conserved among GIY-YIG family members (Garrison \& Arrizabalaga, 2009). We also identified putative mitochondrion-targeted (DeepLoc-1.0L: Mitochondrion 0.9268 and 0.6334, Soluble 0.5699 and 0.6798 ) homologues of RECA proteins, which are also predicted to play a role in controlling mitochondrial genome maintenance in plants. Searches of the $O$. quekettii transcriptome revealed potential OSB1 homologues, predicted to be involved in homologous recombination-dependent repair, containing a central OB-fold domain but lacking a targeting signal to the mitochondrion or chloroplast (DeepLoc-1.0L: Nucleus 0.6485, Soluble $0.7227)$.

\section{Discussion}

The inflated mitochondrial genome of Ostreobium quekettii, the largest Chlorophyta mitochondrial genome sequenced thus far, even larger than that of $C$. lentillifera, lends support 
to large mitochondrial genomes being a characteristic of the order Bryopsidales. The O. quekettii mitochondrial genome encodes all genes commonly found in Chlorophyta mitochondrial genomes, including most ribosomal protein genes, which have been unevenly retained in plant mitochondria (Palmer et al., 2000; Mower et al., 2012), and are missing in some Chlorophyta lineages such as the Chlorophyceae and Pedinomonas (e.g. Figure S3). Surprisingly, although ribosomal proteins have been identified in other Ulvophyceae mitochondrial genomes thus far, none were identified in the mitochondrial genome of $C$. lentillifera (Zheng et al., 2018). The large size of both Bryopsidales mitochondrial genomes relative to their plastids is not typical of the Chlorophyta, where chloroplast genomes tend to be either similar size or larger and contain more noncoding DNA than their mostly compact intron-poor mitochondrial counterparts (Leliaert et al., 2012). Instead, these genomes are more typical of land plants: bloated with introns and intergenic DNA (Leliaert et al., 2012; Mower et al., 2012). In fact, the Bryopsidales mitochondrial genomes are larger than those of many streptophytes, above the average range cited for non-vascular land plants but not quite as extreme as has been described in the Trachaeophytes (Mower et al., 2012). The GC content of the Bryopsidales mitochondrial genomes is also high, markedly higher than their chloroplasts. Differing nucleotide content between organelle genomes, however, is not unheard of in green algae, nor is drastic variation in organelle nucleotide content between even closely related lineages (Smith et al., 2011; Del Vasto et al., 2015).

There is evidence for at least proliferation of type II introns within the Ostreobium lineage, something that is not uncommon in green algal chloroplast genomes (summarised in Cremen et al., 2018). However, a lack of sequence similarity for ORFs identified between their genomes, alongside the fact that their introns are not readily alignable, suggests that either the introns and 
associated proteins arose independently between $C$. lentillifera and $O$. quekettii before proliferating within their respective lineages, or that sequences have diverged so much that similarities are no longer recognisable. This is not unlikely given the estimated 479 million years divergence time between these lineages (Verbruggen et al., 2009). Cremen et al. (2018) did find some homologous ORFs, with conserved protein domains, between Bryopsidales chloroplast genomes. The lack of similarity between Ostreobium mitochondrial ORFs and its single plastid ORF with a putative intron splicing function (Cremen et al., 2018), as well as between introns from the organellar genomes, indicates there has been no transfer of introns between organellar genomes.

The considerable difference in their sizes and gene density as well as a lack of evidence for ORF or intron transfer between organellar compartments indicates that evolutionary forces are acting upon plastid and mitochondrial genomes differently in the Bryopsidales. The mutational-hazard (MHH) (Lynch et al., 2006) and drift-barrier (Lynch et al., 2016) hypotheses emphasise the importance of mutation rate in determining genome sizes. Molecular evolution rates of the Ostreobium chloroplast are slow compared with other Bryopsidales; Marcelino et al. (2016) propose that this is due to the low light habitat of Ostreobium, which might reduce cases of direct sunlight-induced mutation as well as drive the evolution of slow metabolic rates and generation times with fewer mutations accumulated per unit of time. Estimates in this study indicate a slightly higher substitution rate in Bryopsidales mitochondrial genomes compared with the chloroplasts, which appears to contradict the MHH that states genomes with a higher mutation rate should show signs of compaction, as they are under greater selective pressure to remove excess DNA that carries a mutational burden (Lynch et al., 2006). $d_{S}$ values estimated from gene alignments show signs of saturated divergence, making accurate calculation of $d_{N} / d_{S}$ unrealistic 
429 (Yang \& Bielawski, 2000; Hurst, 2002). This is unsurprising due to the considerable time since 430 the divergence of these lineages. It is not uncommon for mutation rates to vary between lineages 431 and even between genome compartments of the same lineages by a considerably larger margin 432 than was estimated in this study, and it can be difficult to draw direct connections between

mutation rates and genome architecture (Smith, 2016). An example is the green alga Dunaliella salina, which contains inflated organelle genomes, both chloroplast and mitochondrial, but has order-of-magnitude differences in mutation rates between the two compartments, with substitution rates between two strains of $D$. salina 2-13 times greater in mtDNA than ptDNA (Del Vasto et al., 2015). Differing mutation rates alone appear insufficient to explain the genome dynamics of the two Bryopsidales organellar compartments.

The higher mutation rate in the Bryopsidales mitochondrial genomes may have driven selection for more efficient DNA repair proteins, as would be predicted by the drift-barrier hypothesis (Lynch et al., 2016). It has been proposed that two distinct strategies have evolved to protect organelle genomes from the negative effects of non-homologous recombination. In animal mtDNA, elevated rates of evolution cause non-coding elements to pose a high mutational burden, leading to selection against non-coding elements, avoiding the build-up of repeats and introns (Lynch et al., 2006; Galtier, 2011). Plants appear to have efficient recombinationmediated DNA repair of coding DNA, explaining their low measured mutation rate (Odahara $e t$ al., 2009; Davila et al., 2011; Christensen, 2014). The nuclear-encoded RECA3 and MSH1 genes in plants are hypothesised to control mitochondrial genome maintenance, by preventing replication of short repeats while allowing recombination-dependent replication of longer repeats (Shedge et al., 2007). We identified a putative mitochondrion-targeted MSH1 homologue in $O$. quekettii. MSH1 encodes a protein with six conserved domains (Kowalski et al., 1999) including 
452 domain VI, a GIY-YIG homing endonuclease, which is predicted to be responsible for specific 453 DNA-binding and suppression of homologous recombination (Fukui et al., 2018) and is specific 454 to only the plant form of the protein (Abdelnoor et al., 2006; Shedge et al., 2007). Domain VI is 455 absent from nuclear localized homologues in plants (MSH2-MSH6) and from the yeast MSH1 456 protein (Abdelnoor et al., 2006). Although InterPro and Pfam predicted a GIY-YIG domain in 457 the $O$. quekettii MSH1 homologue, the fact that it lacks most of the residues that are typically 458 conserved in this domain leaves its function unresolved.

O. quekettii also appears to encode mitochondrially-targeted RecA proteins. RecA recombinases in Arabidopsis are involved in strand exchange and the joining of paired DNA ends during homologous recombination (Kühn \& Gualberto, 2012; Gualberto et al., 2014). RECA1 is chloroplast targeted, RECA2 is dual targeted to the mitochondrion and chloroplast, and RECA3 is targeted to the mitochondrion (Shedge et al., 2007). RECA3 mutations in Arabidopsis result in mitochondrial rearrangements similar but not identical to MSH1 mutants (Shedge et al., 2007). Odahara et al. (2009) propose that these RecA proteins mediate homologous recombination, which is significant for suppressing short repeat-mediated genome rearrangements in plant mitochondria. They suggest that this genome stabilisation provided by RecA could allow the number of group II introns, the dominant form in the O. quekettii mitochondrion, to increase (Odahara et al., 2009). OSB1 is another putative component of homologous recombinationdependent repair in plant mitochondria, which is also likely involved in restricting mtDNA recombination (Kühn \& Gualberto, 2012; Gualberto et al., 2014). Searches of the O. quekettii transcriptome revealed potential OSB1 homologues. However, these lack targeting signals to organelles that would provide evidence supporting their predicted function. 
474 In contrast to the mitochondrial and chloroplast genomes of Volvox carteri (Smith \& Lee, 2009),

475 and those recently published for Haematococcus lacustris (Zhang et al., 2019), as well as the

476 mitochondrial genomes of many land plants (Palmer et al., 2000; Mower et al., 2012), little of

477 the expanded content of Bryopsidales mtDNA is repetitive DNA. However, most of the repeats

478 in the O. quekettii mitochondrial genome are so-called 'intermediate' repeats (50-600 bp) (Kühn

$479 \&$ Gualberto, 2012). Repeats of this length are associated with MSH1-induced recombination in

480 Arabidopsis mitochondria, that can lead to accumulation of DNA as well as complex

481

482

483

rearrangements (Gualberto et al., 2014). Along with error prone repair these processes might result in low numbers of alternative genome configurations, 'mitotypes' that could eventually increase to become the dominant form of mtDNA (Gualberto et al., 2014). This working hypothesis, based on molecular data, proposes that a recombination-associated repair process in the Bryopsidales mitochondrion has resulted in its inflated size, and encourages further study to resolve the role played by these putative mitochondrion-targeted sequences as well as to determine whether recombination is in fact occurring in Bryopsidales mitochondrial genomes at all.

It is interesting to consider whether the buildup of introns in Bryopsidales mitochondrial genomes plays a part in modifying recombination if it is in fact occurring. Introns may play a role in reducing intragenic Hill-Roberston effects, that tend to result in locally reduced $N_{e}$, by increasing recombination rate between regions of the genome (Comeron \& Kreitman, 2000). Bottlenecking of genomes, such as during the production of gametes for sexual reproduction, can also result in smaller genome $N_{e}$ (Neiman \& Taylor, 2009), which would reduce selection and might contribute to the higher mutational load observed in Bryopsidales mitochondrial genomes. Sexual reproduction has not been observed in $O$. quekettii, however it has been described in 
497 other Bryopsidales (e.g. Morabito et al., 2010) and, to our knowledge, no strictly asexual

498 lineages have been observed. Comparing relative copy numbers of organelle genomes between

499 adult plants and gametes could provide insight into the extent of bottlenecking that occurs during

500 reproduction in the Bryopsidales.

501 The mutation and recombination rates in organelles are at least in part under the control of

502 nuclear-encoded maintenance pathways (Smith \& Keeling, 2015), with organelle genomes

503 usually lacking the genes necessary for their own DNA replication and repair (Sloan \& Taylor,

504 2012). It has been proposed that the evolution of similar features in both plastid and

505 mitochondrial genomes within a species, as seen in some of the Chlorophyta, is due to 'leakage'

506 of nuclear-encoded proteins controlling these processes between organellar compartments, with

507 proteins normally targeted to one organelle also becoming targeted to the other (Smith \&

508 Keeling, 2015). The differing configurations of the two organellar genomes in Caulerpa

509 lentillifera and Ostreobium quekettii suggest that this has not occurred in the Bryopsidales. As

510 more nuclear genomes for Bryopsidales become available (e.g. Arimoto et al., 2019), there will

511 soon be the opportunity for a more thorough study of all three genomic compartments in the

512 Bryopsidales, including an examination of organelle-targeted DNA maintenance machinery to

513 further uncover the forces underpinning their divergent organelle genome sizes.

\section{Conclusions}

Marcelino et al. (2016) suggested that selection due to a low light environment resulted in the

517 reduced chloroplast genome of $O$. quekettii, but it is unclear why this selection would not act against the expanded genome size in the mitochondrion. It would appear that effective 
519 population size, recombination and/or mutation rate, influenced by nuclear-encoded proteins, are 520 different between the two genomes, leading to a reduction in the strength of selection to 521 influence evolution of the mitochondrial genome. Ultimately, it is likely overly simplistic to 522 attempt to find a single explanation to cover all mitochondrial genome expansion (Smith \& 523 Keeling, 2015), with the evolution of organellar genomes in the Bryopsidales and other lineages 524 a combination of many forces and factors.

\section{Acknowledgements}

This research was supported by use of the computational facilities of the University of Melbourne High Performance Computing platform and the Nectar Research Cloud. This paper benefitted from useful discussions with Joseph Bielawski about evolution, as well as helpful comments from Geoffrey McFadden and Patrick Buerger on an earlier draft. We appreciate the thoughtful comments on the manuscript made by Jiri Neustupa and an anonymous reviewer.

\section{References}

Abdelnoor RV, Christensen AC, Mohammed S, Munoz-Castillo B, Moriyama H, Mackenzie SA. 2006. Mitochondrial genome dynamics in plants and animals: convergent gene fusions of a MutS homologue. Journal of Molecular Evolution 63: 165-173.

Abe T, Ikemura T, Sugahara J, Kanai A, Ohara Y, Uehara H, Kinouchi M, Kanaya S, Yamada Y, Muto A. 2010. tRNADB-CE 2011: tRNA gene database curated manually by experts. Nucleic Acids Research 39: D210-D213.

Alkatib S, Scharff LB, Rogalski M, Fleischmann TT, Matthes A, Seeger S, Schöttler MA, Ruf S, Bock R. 2012. The contributions of wobbling and superwobbling to the reading of the genetic code. PLoS Genetics 8: e1003076. 
543 Almagro Armenteros JJ, Sønderby CK, Sønderby SK, Nielsen H, Winther O. 2017. DeepLoc:

544 prediction of protein subcellular localization using deep learning. Bioinformatics 33: 3387-3395.

545 Arimoto A, Nishitsuji K, Higa Y, Arakaki N, Hisata K, Shinzato C, Satoh N, Shoguchi E. 2019.

546 A siphonous macroalgal genome suggests convergent functions of homeobox genes in algae and

547 land plants. DNA Research 26: 183-192.

548 Bankevich A, Nurk S, Antipov D, Gurevich AA, Dvorkin M, Kulikov AS, Lesin VM, Nikolenko

549 SI, Pham S, Prjibelski AD. 2012. SPAdes: a new genome assembly algorithm and its

550 applications to single-cell sequencing. Journal of Computational Biology 19: 455-477.

551 Beck N, Lang B. 2010. MFannot, organelle genome annotation websever. Canada: Université de 552 Montréal QC.

553 Bolger AM, Lohse M, Usadel B. 2014. Trimmomatic: a flexible trimmer for Illumina sequence 554 data. Bioinformatics 30: 2114-2120.

555 Burger G, Gray MW, Lang BF. 2003. Mitochondrial genomes: anything goes. Trends in 556 Genetics 19: 709-716.

557 Carrie C, Weißenberger S, Soll J. 2016. Plant mitochondria contain the protein translocase 558 subunits TatB and TatC. Journal of Cell Science 129: 3935-3947.

559 Charlesworth B. 1994. The effect of background selection against deleterious mutations on 560 weakly selected, linked variants. Genetics Research 63(3):213-227.

561 Christensen AC. 2014. Genes and junk in plant mitochondria-repair mechanisms and selection. 562 Genome Biology and Evolution 6: 1448-1453. 
563 Cocquyt E, Verbruggen H, Leliaert F, De Clerck O. 2010. Evolution and cytological

564 diversification of the green seaweeds (Ulvophyceae). Molecular Biology and Evolution 27:

$565 \quad 2052-2061$.

566 Comeron JM, Kreitman M. 2000. The correlation between intron length and recombination in 567 Drosophila: dynamic equilibrium between mutational and selective forces. Genetics 156: 11755681190.

569 Comeron JM, Williford A, Kliman R. 2008. The Hill-Robertson effect: evolutionary 570 consequences of weak selection and linkage in finite populations. Heredity 100: 19.

571 Cremen MCM, Huisman JM, Marcelino VR, Verbruggen H. 2016. Taxonomic revision of 572 Halimeda (Bryopsidales, Chlorophyta) in south-western Australia. Australian Systematic Botany $573 \quad 29: 41-54$.

574 Cremen MCM, Leliaert F, Marcelino VR, Verbruggen H. 2018. Large Diversity of Nonstandard 575 Genes and Dynamic Evolution of Chloroplast Genomes in Siphonous Green Algae 576 (Bryopsidales, Chlorophyta). Genome Biology and Evolution 10: 1048-1061.

577 Cutter AD, Payseur BA. 2013. Genomic signatures of selection at linked sites: unifying the 578 disparity among species. Nature Reviews Genetics 14: 262.

579 580 581

Davila JI, Arrieta-Montiel MP, Wamboldt Y, Cao J, Hagmann J, Shedge V, Xu Y-Z, Weigel D, Mackenzie SA. 2011. Double-strand break repair processes drive evolution of the mitochondrial genome in Arabidopsis. BMC Biology 9: 64.

de Vries J, Habicht J, Woehle C, Huang C, Christa G, Wägele H, Nickelsen J, Martin WF, Gould SB. 2013. Is ftsH the key to plastid longevity in sacoglossan slugs? Genome Biology and Evolution 5: 2540-2548. 
585 586

587 588

589 590

591

592

593

594

595

596

597

598

599

600

601

602

603

604 605

Del Cortona A, Leliaert F, Bogaert KA, Turmel M, Boedeker C, Janouškovec J, Lopez-Bautista JM, Verbruggen H, Vandepoele K, De Clerck O. 2017. The plastid genome in Cladophorales green algae is encoded by hairpin chromosomes. Current Biology 27: 3771-3782.

Del Vasto M, Figueroa-Martinez F, Featherston J, Gonzalez MA, Reyes-Prieto A, Durand PM, Smith DR. 2015. Massive and widespread organelle genomic expansion in the green algal genus Dunaliella. Genome Biology and Evolution 7: 656-663.

Fang L, Leliaert F, Zhang ZH, Penny D, Zhong BJ. 2017. Evolution of the Chlorophyta: Insights from chloroplast phylogenomic analyses. Journal of Systematics and Evolution 55: 322-332.

Felsenstein, J. 2005. PHYLIP (Phylogeny Inference Package) version 3.6. Distributed by the author. Department of Genome Sciences, University of Washington, Seattle.

Finn RD, Attwood TK, Babbitt PC, Bateman A, Bork P, Bridge AJ, Chang H-Y, Dosztányi Z, El-Gebali S, Fraser M. 2016. InterPro in 2017-beyond protein family and domain annotations. Nucleic Acids Research 45: D190-D199.

Frickey T, Lupas A. 2004. CLANS: a Java application for visualizing protein families based on pairwise similarity. Bioinformatics 20: 3702-3704.

Fučíková K, Leliaert F, Cooper ED, Škaloud P, D'hondt S, De Clerck O, Gurgel CF, Lewis LA, Lewis PO, Lopez-Bautista JM. 2014. New phylogenetic hypotheses for the core Chlorophyta based on chloroplast sequence data. Frontiers in Ecology and Evolution 2: 63.

Fukui K, Harada A, Wakamatsu T, Minobe A, Ohshita K, Ashiuchi M, Yano T. 2018. The GIY-YIG endonuclease domain of Arabidopsis MutS homolog 1 specifically binds to branched DNA structures. FEBS Letters 592: 4066-4077. 
606 Galtier N. 2011. The intriguing evolutionary dynamics of plant mitochondrial DNA. BMC

607 Biology 9: 61.

608 Garrison EM, Arrizabalaga G. 2009. Disruption of a mitochondrial MutS DNA repair enzyme

609 homologue confers drug resistance in the parasite Toxoplasma gondii. Molecular Microbiology

$610 \quad 72: 425-441$.

611 Gelfand Y, Rodriguez A, Benson G. 2006. TRDB - the tandem repeats database. Nucleic Acids

612 Research 35: D80-D87.

613 Grabherr MG, Haas BJ, Yassour M, Levin JZ, Thompson DA, Amit I, Adiconis X, Fan L,

614 Raychowdhury R, Zeng Q. 2011. Full-length transcriptome assembly from RNA-Seq data

615 without a reference genome. Nature Biotechnology 29: 644.

616 Griffiths-Jones S, Bateman A, Marshall M, Khanna A, Eddy SR. 2003. Rfam: an RNA family

617 database. Nucleic Acids Research 31: 439-441.

618 Gualberto JM, Mileshina D, Wallet C, Niazi AK, Weber-Lotfi F, Dietrich A. 2014. The plant

619 mitochondrial genome: dynamics and maintenance. Biochimie 100: 107-120.

620 Hausner G 2012. Introns, mobile elements, and plasmids. In: Bullerwell CE, eds. Organelle

621 Genetics. Berlin, Germany: Springer, 329-357.

622 Hill WG, Robertson A. 1966. The effect of linkage on limits to artificial selection. Genetics

623 Research 8: 269-294.

624 Hurst LD. 2002. The Ka/Ks ratio: diagnosing the form of sequence evolution. Trends in 625 genetics: TIG 18: 486-486.

626 Jukes TH, Cantor CR. 1969. Evolution of protein molecules. Mammalian Protein Metabolism 3: $627 \quad 132$. 
628 Kearse M, Moir R, Wilson A, Stones-Havas S, Cheung M, Sturrock S, Buxton S, Cooper A, 629 Markowitz S, Duran C. 2012. Geneious Basic: an integrated and extendable desktop software 630 platform for the organization and analysis of sequence data. Bioinformatics 28: 1647-1649.

631 Kinouchi M, Kurokawa K. 2006. tRNAfinder: a software system to find all tRNA genes in the 632 DNA sequence based on the cloverleaf secondary structure. Journal of Computer Aided 633 Chemistry 7: 116-126.

634 Koren S, Walenz BP, Berlin K, Miller JR, Bergman NH, Phillippy AM. 2017. Canu: scalable 635 and accurate long-read assembly via adaptive k-mer weighting and repeat separation. Genome 636 Research 27: 722-736.

637 Kowalski JC, Belfort M, Stapleton MA, Holpert M, Dansereau JT, Pietrokovski S, Baxter SM, 638 Derbyshire V. 1999. Configuration of the catalytic GIY-YIG domain of intron endonuclease I639 Tev I: coincidence of computational and molecular findings. Nucleic Acids Research 27: $2115-$ 6402125.

641 Krasovec M, Eyre-Walker A, Sanchez-Ferandin S, Piganeau G. 2017. Spontaneous mutation rate 642 in the smallest photosynthetic eukaryotes. Molecular Biology and Evolution 34: 1770-1779.

643 Krzywinski M, Schein J, Birol I, Connors J, Gascoyne R, Horsman D, Jones SJ, Marra MA. 644 2009. Circos: an information aesthetic for comparative genomics. Genome Research 19: 16396451645.

646 Kumar S, Stecher G, Li M, Knyaz C, Tamura K. 2018. MEGA X: molecular evolutionary 647 genetics analysis across computing platforms. Molecular Biology and Evolution 35: 1547-1549. 
648 Kurtz S, Choudhuri JV, Ohlebusch E, Schleiermacher C, Stoye J, Giegerich R. 2001. REPuter:

649 the manifold applications of repeat analysis on a genomic scale. Nucleic Acids Research 29:

$650 \quad 4633-4642$.

651 Kühn K, Gualberto JM 2012. Recombination in the stability, repair and evolution of the

652 mitochondrial genome. In: Maréchal-Drouard L, ed. Advances in Botanical Research 63.

653 Oxford, UK: Academic Press, 215-252.

654 Lagesen K, Hallin P, Rødland EA, Stærfeldt H-H, Rognes T, Ussery DW. 2007. RNAmmer:

655 consistent and rapid annotation of ribosomal RNA genes. Nucleic Acids Research 35: 3100-

6563108.

657 Lambowitz AM, Belfort M. 1993. Introns as mobile genetic elements. Annual Review of

658 Biochemistry 62: 587-622.

659

660

661

662

663

664

665

666

667

668

669

Lambowitz AM, Zimmerly S. 2011. Group II introns: mobile ribozymes that invade DNA. Cold Spring Harbor Perspectives in Biology 3: a003616.

Lang BF, Laforest M-J, Burger G. 2007. Mitochondrial introns: a critical view. Trends in Genetics 23: 119-125.

Laslett D, Canback B. 2004. ARAGORN, a program to detect tRNA genes and tmRNA genes in nucleotide sequences. Nucleic Acids Research 32: 11-16.

Leliaert F, Smith DR, Moreau H, Herron MD, Verbruggen H, Delwiche CF, De Clerck O. 2012. Phylogeny and molecular evolution of the green algae. Critical Reviews In Plant Sciences 31: 146.

Lowe TM, Chan PP. 2016. tRNAscan-SE On-line: integrating search and context for analysis of transfer RNA genes. Nucleic Acids Research 44: W54-W57. 
670 Lynch M. 2006. Streamlining and simplification of microbial genome architecture. Annual 671 Review of Microbiology 60: 327-349.

672 Lynch M, Ackerman MS, Gout J-F, Long H, Sung W, Thomas WK, Foster PL. 2016. Genetic 673 drift, selection and the evolution of the mutation rate. Nature Reviews Genetics 17: 704.

674 Lynch M, Koskella B, Schaack S. 2006. Mutation pressure and the evolution of organelle 675 genomic architecture. Science 311: 1727-1730.

676 Lynch M, Walsh B. 2007. The origins of genome architecture 98. Sunderland, MA: Sinauer 677 Associates.

Magnusson SH, Fine M, Kühl M. 2007. Light microclimate of endolithic phototrophs in the scleractinian corals Montipora monasteriata and Porites cylindrica. Marine Ecology Progress Series 332: 119-128.

Marcelino V, Cremen MCM, Jackson CJ, Larkum AA, Verbruggen H. 2016. Evolutionary dynamics of chloroplast genomes in low light: a case study of the endolithic green alga Ostreobium quekettii. Genome Biology and Evolution 8: 2939-2951.

Martin M. 2011. Cutadapt removes adapter sequences from high-throughput sequencing reads. EMBnet.journal 17: 10-12.

Mine I, Sekida S, Okuda K. 2015. Cell wall and cell growth characteristics of giant-celled algae. Phycological Research 63: 77-84.

Morabito M, Gargiulo G, Genovese G. 2010. A review of life history pathways in Bryopsis. Atti della Accademia Peloritana dei Pericolanti-Classe di Scienze Fisiche, Matematiche e Naturali 88. doi: 10.1478/C1A1002005 
691 Mower JP, Sloan DB, Alverson AJ 2012. Plant mitochondrial genome diversity: the genomics

692 revolution. In: Wendel J, Greilhuber J, Dolezel J, Leitch IJ, eds. Plant Genome Diversity

693 Volume 1. Wien, Austria: Springer, 123-144

694 Neiman M, Taylor DR. 2009. The causes of mutation accumulation in mitochondrial genomes.

695 Proceedings of the Royal Society B: Biological Sciences 276:1201-1209.

696 Ness RW, Kraemer SA, Colegrave N, Keightley PD. 2015. Direct estimate of the spontaneous

697 mutation rate uncovers the effects of drift and recombination in the Chlamydomonas reinhardtii

698 plastid genome. Molecular Biology and Evolution 33: 800-808.

699 Odahara M, Kuroiwa H, Kuroiwa T, Sekine Y. 2009. Suppression of repeat-mediated gross

700 mitochondrial genome rearrangements by RecA in the moss Physcomitrella patens. The Plant

701 Cell 21: 1182-1194.

702 Palmer JD, Adams KL, Cho Y, Parkinson CL, Qiu Y-L, Song K. 2000. Dynamic evolution of

703 plant mitochondrial genomes: mobile genes and introns and highly variable mutation rates.

704 Proceedings of the National Academy of Sciences 97: 6960-6966.

705 Petrů M, Wideman J, Moore K, Alcock F, Palmer T, Doležal P. 2018. Evolution of

706 mitochondrial TAT translocases illustrates the loss of bacterial protein transport machines in

707 mitochondria. BMC Biology 16: 141.

708 Platt A, Weber CC, Liberles DA. 2018. Protein evolution depends on multiple distinct

709 population size parameters. BMC Evolutionary Biology 18: 17.

710 Pombert J-F, Otis C, Lemieux C, Turmel M. 2004. The complete mitochondrial DNA sequence

711 of the green alga Pseudendoclonium akinetum (Ulvophyceae) highlights distinctive evolutionary 
712 trends in the Chlorophyta and suggests a sister-group relationship between the Ulvophyceae and 713 Chlorophyceae. Molecular Biology and Evolution 21: 922-935.

714 Ricci F, Marcelino VR, Blackall L, Kühl M, Medina M, Verbruggen H. 2019. Beneath the 715 surface: community assembly and functions of the coral skeleton microbiome. EcoEvoRxiv: 716 DOI: 10.32942/osf.io/9yjw8.

717 Schlichter D, Kampmann H, Conrady S. 1997. Trophic potential and photoecology of endolithic 718 algae living within coral skeletons. Marine Ecology 18: 299-317.

719 Shedge V, Arrieta-Montiel M, Christensen AC, Mackenzie SA. 2007. Plant mitochondrial 720 recombination surveillance requires unusual RecA and MutS homologs. The Plant Cell 19: $721 \quad 1251-1264$.

722 Sievers F, Wilm A, Dineen D, Gibson TJ, Karplus K, Li W, Lopez R, McWilliam H, Remmert 723 M, Söding J. 2011. Fast, scalable generation of high-quality protein multiple sequence 724 alignments using Clustal Omega. Molecular Systems Biology 7: 539.

725 Sloan DB, Taylor DR 2012. Evolutionary rate variation in organelle genomes: the role of 726 mutational processes. In: Bullerwell CE, eds. Organelle Genetics. Berlin, Germany: Springer, $727 \quad 123-146$.

728 Smith D, Lee RW. 2009. The mitochondrial and plastid genomes of Volvox carteri: bloated 729 molecules rich in repetitive DNA. BMC Genomics 10: 1.

730 Smith DR. 2016. The mutational hazard hypothesis of organelle genome evolution: 10 years on. 731 Molecular Ecology 25: 3769-3775. 
732 Smith DR, Burki F, Yamada T, Grimwood J, Grigoriev IV, Van Etten JL, Keeling PJ. 2011. The 733 GC-rich mitochondrial and plastid genomes of the green alga Coccomyxa give insight into the 734 evolution of organelle DNA nucleotide landscape. PLoS One 6:e23624.

735 Smith DR, Hamaji T, Olson BJ, Durand PM, Ferris P, Michod RE, Featherston J, Nozaki H, 736 Keeling PJ. 2013. Organelle genome complexity scales positively with organism size in 737 volvocine green algae. Molecular Biology and Evolution 30: 793-797.

738 Smith DR, Keeling PJ. 2015. Mitochondrial and plastid genome architecture: reoccurring 739 themes, but significant differences at the extremes. Proceedings of the National Academy of 740 Sciences 112: 10177-10184.

741 Smith DR, Lee RW. 2007. Mitochondrial genome of the colorless green alga Polytomella 742 capuana: a linear molecule with an unprecedented GC content. Molecular Biology and Evolution $743 \quad 25: 487-496$.

744 Smith DR, Lee RW. 2010. Low nucleotide diversity for the expanded organelle and nuclear 745 genomes of Volvox carteri supports the mutational-hazard hypothesis. Molecular Biology and 746 Evolution 27: 2244-2256.

747 Smith DR, Lee RW, Cushman JC, Magnuson JK, Tran D, Polle JE. 2010. The Dunaliella salina 748 organelle genomes: large sequences, inflated with intronic and intergenic DNA. BMC Plant 749 Biology 10: 83.

750 Smith JM, Haigh J. 1974. The hitch-hiking effect of a favourable gene. Genetics Research 23: $751 \quad 23-35$.

752 Suyama M, Torrents D, Bork P. 2006. PAL2NAL: robust conversion of protein sequence 753 alignments into the corresponding codon alignments. Nucleic Acids Research 34: W609-W612. 
754 Tribollet A 2008. The boring microflora in modern coral reef ecosystems: a review of its roles.

755 In Wisshak M, Tapanila L, eds. Current Developments in Bioerosion. Berlin, Heidelberg:

756 Springer, 67-94.

757 Verbruggen H, Ashworth M, LoDuca ST, Vlaeminck C, Cocquyt E, Sauvage T, Zechman FW, 758 Littler DS, Littler MM, Leliaert F. 2009. A multi-locus time-calibrated phylogeny of the 759 siphonous green algae. Molecular Phylogenetics and Evolution 50: 642-653.

760 Verbruggen H, Marcelino VR, Guiry MD, Cremen MCM, Jackson CJ. 2017. Phylogenetic 761 position of the coral symbiont Ostreobium (Ulvophyceae) inferred from chloroplast genome 762 data. Journal of Phycology 53: 790-803.

763 Verbruggen H, Tribollet A. 2011. Boring algae. Current Biology 21: R876-R877.

764 Wilhelm C, Jakob T. 2006. Uphill energy transfer from long-wavelength absorbing chlorophylls 765 to PS II in Ostreobium sp. is functional in carbon assimilation. Photosynthesis Research 87: 323.

766 Vroom PS, Smith CM. 2001. The challenge of siphonous green algae. American Scientist 89: $767 \quad 525-531$.

768 Vroom PS, Smith CM. 2003. Life without cells. Biologist 50: 222-226.

769 Wyman SK, Jansen RK, Boore JL. 2004. Automatic annotation of organellar genomes with 770 DOGMA. Bioinformatics 20: 3252-3255.

771 Yang Z, Bielawski JP. 2000. Statistical methods for detecting molecular adaptation. Trends in 772 Ecology \& Evolution 15: 496-503.

773 Yurina N, Odintsova M. 2016. Mitochondrial genome structure of photosynthetic eukaryotes. 774 Biochemistry (Moscow) 81: 101-113. 
775 Zhang X, Bauman N, Brown R, Richardson TH, Akella S, Hann E, Morey R, Smith DR. 2019.

776 The mitochondrial and chloroplast genomes of the green alga Haematococcus are made up of

777 nearly identical repetitive sequences. Current Biology 29: R736-R737.

778 Zheng F, Liu H, Jiang M, Xu Z, Wang Z, Wang C, Du F, Shen Z, Wang B. 2018. The complete

779 mitochondrial genome of the Caulerpa lentillifera (Ulvophyceae, Chlorophyta): Sequence,

780 genome content, organization structure and phylogenetic consideration. Gene 673: 225-238.

781 Zimin AV, Marçais G, Puiu D, Roberts M, Salzberg SL, Yorke JA. 2013. The MaSuRCA

782 genome assembler. Bioinformatics 29: 2669-2677.

783 Zimmermann L, Stephens A, Nam S-Z, Rau D, Kübler J, Lozajic M, Gabler F, Söding J, Lupas

784 AN, Alva V. 2018. A completely Reimplemented MPI bioinformatics toolkit with a new HHpred

785 server at its Core. Journal of Molecular Biology 430: 2237-2243. 


\section{Figure 1}

Mitochondrial genome map of Ostreobium quekettii SAG6.99

The position of tRNAs are shown on the outer track (red lines). The first inner circle represents the position, size and the names of the protein-coding and rRNA genes. The introns are shown in the second inner circle and are colour coded according to intron types/subtypes: group I derived (very light blue), group IA (light blue), group IB (blue), group ID (dark blue), group II (orange), unknown (grey). The third inner circle represents the position and length (line thickness) of repeats. 


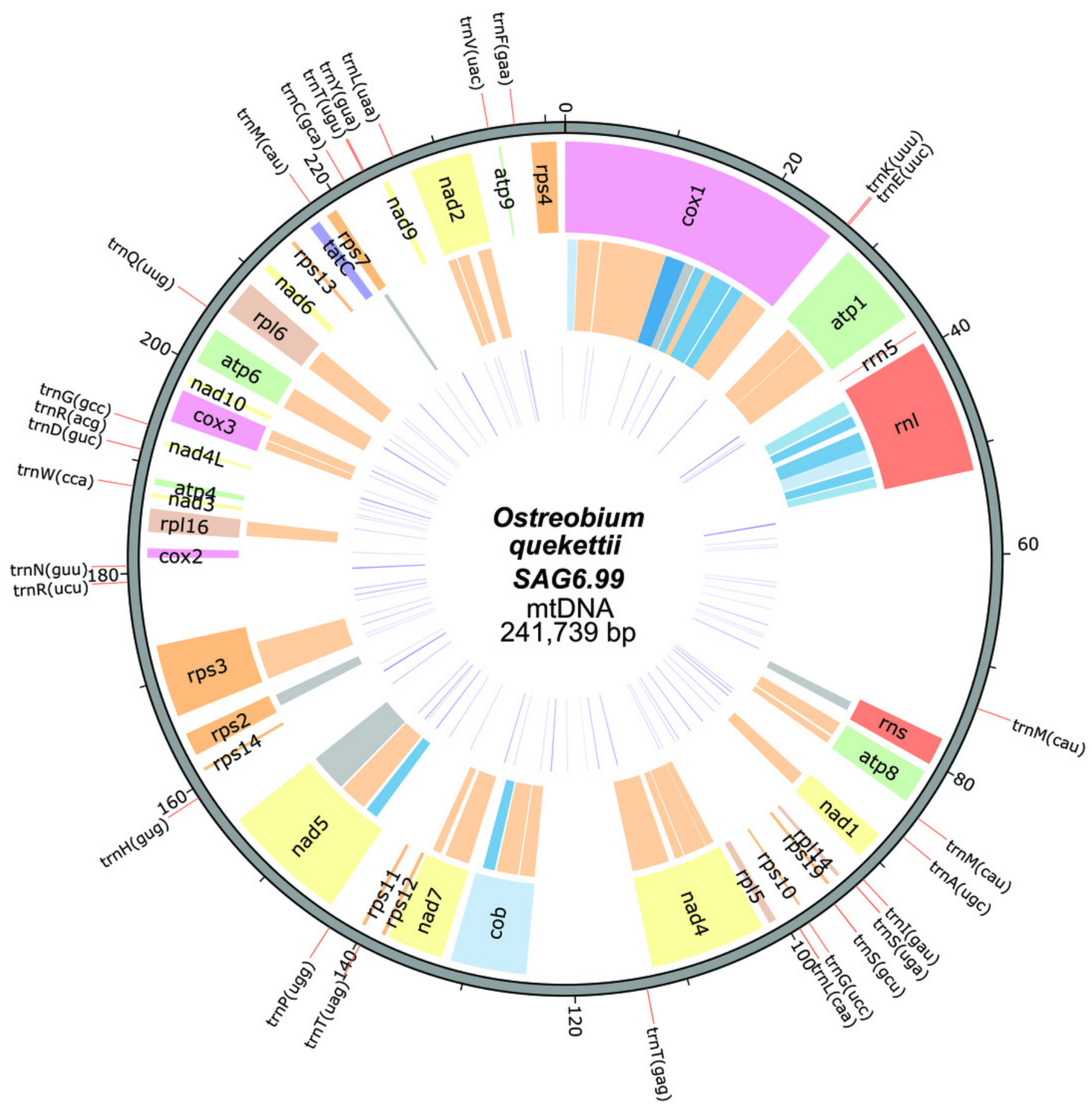


Figure 2

Similarity network generated from all against all BLAST+ similarities of ORFs encoded in the Caulerpa lentillifera mitochondrion, and Ostreobium quekettii mitochondrion and chloroplast.

Each node represents an ORF, and each edge (line) represents a significant HSP (high scoring segment pair), shaded according to $p$ value. Generated using CLANS through the MPI Bioinformatics Toolkit (Scoring Matrix BLOSSUM62, extracting BLAST HSPs up to E-values of 1e-4, using p-values better than 1.0).

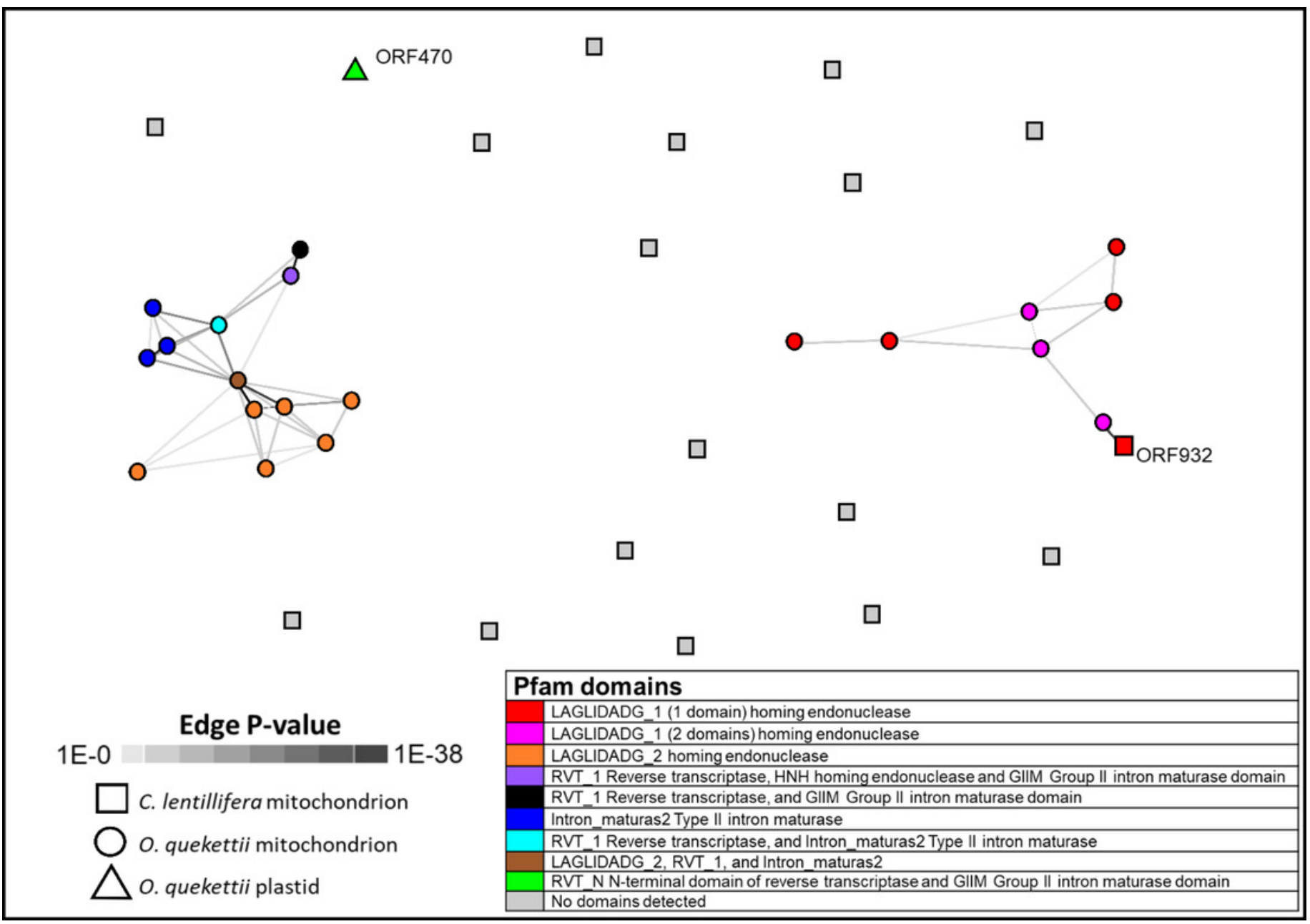




\section{Table $\mathbf{1}$ (on next page)}

Summary of coding and noncoding content of the mtDNA of Ostreobium quekettii SAG6.99. 


\begin{tabular}{|lrr|}
\hline & $\begin{array}{l}\text { Percent of total } \\
\text { noncoding DNA }\end{array}$ & $\begin{array}{l}\text { Percent of overall } \\
\text { genome }\end{array}$ \\
Coding (rRNA, tRNA, ORFs, protein coding genes) & $7 \%$ & $5 \%$ \\
\hline Repeats & $61 \%$ & $46 \%$ \\
\hline Intergenic DNA & & $39 \%$ \\
\hline Introns (including ORFs) & $39 \%$ & $29 \%$ \\
\hline Introns (excluding ORFs) & & $75 \%$ \\
\hline Total noncoding DNA (excluding ORFs) & $85 \%$ \\
\hline Total intronic and intergenic DNA (including ORFs) & & $5 \%$ \\
\hline
\end{tabular}

1 


\section{Table 2 (on next page)}

Genes, introns and open reading frames present in mtDNA of Ostreobium quekettii SAG6.99. For further information on introns and ORFs, see supplementary material. 
1

\begin{tabular}{|lr|}
\hline & $\begin{array}{r}\text { Number in } \\
\text { genome }\end{array}$ \\
\hline Protein coding genes & 34 \\
\hline rRNA & 3 \\
\hline tRNA & 28 \\
\hline Genes containing introns & 18 \\
\hline Introns & 47 \\
Type I & 14 \\
Type 2 & 28 \\
unclear & 5 \\
\hline Introns containing ORFs & 18 \\
\hline ORFS & 20 \\
\hline \multicolumn{2}{r}{ Intronic } \\
\hline \multicolumn{2}{r}{ Intergenic } \\
\hline
\end{tabular}




\section{Table 3(on next page)}

Protein coding and ribosomal genes present in the mtDNA of Ostreobium quekettii SAG6.99. 


\begin{tabular}{|ll|}
\hline Protein genes & \\
\hline Complex I (nad) & $n a d 1, n a d 2, n a d 3, n a d 4, n a d 4 \mathrm{~L}$, nad5, nad6, nad7, nad9, nad10 \\
\hline Complex III (cob) & $c o b$ \\
\hline Complex IV (cox) & $c o x 1, \operatorname{cox} 2, \operatorname{cox} 3$ \\
\hline Complex V (atp) & $a t p 1, a t p 4, a t p 6, a t p 8, a t p 9$ \\
\hline SSU ribosomal proteins $(\boldsymbol{r p s})$ & $r p s 2, r p s 3, r p s 4, r p s 7, r p s 10, r p s 11, r p s 12, r p s 13, r p s 14, r p s 19$ \\
\hline LSU ribosomal proteins $(\boldsymbol{r p l})$ & $r p l 5, r p l 6, r p l 14, r p l 16$ \\
\hline Ribosomal RNAs & $r r n 5, r r n S, r r n \mathrm{~L}$ \\
\hline Putative Protein Transporter & $t a t C$ \\
\hline
\end{tabular}

